\title{
Preface to JAISE 13(5)
}

\author{
Andrés Muñoz ${ }^{\mathrm{a}}$, Juan Carlos Augusto ${ }^{\mathrm{b}}$, Vincent Tam ${ }^{\mathrm{c}}$ and Hamid Aghajan ${ }^{\mathrm{d}}$ \\ ${ }^{a}$ Polytechnic School, Catholic University of Murcia, Spain \\ ${ }^{\mathrm{b}}$ Department of Computer Science and Research Group on Development of Intelligent Environments, Middlesex \\ University, $U K$ \\ ${ }^{\mathrm{c}}$ Department of Electrical and Electronic Engineering, Faculty of Engineering, the University of Hong Kong, \\ China \\ $\mathrm{d}$ imec, IPI, Department of Telecommunications and Information Processing, Gent University, Belgium
}

\section{This issue}

This regular issue of JAISE is composed of four articles. The review process for the articles in this issue was supervised by our editors Chen Chen, Vincent Tam and Neeraj Kumar (invited editor), whom we thank for their service.

Deep Learning (DL) and Visual Analytics (VA) are two important focus areas serving critical applications in cybersecurity, fraud detection, and many other underlying decision-making processes involved in the operation of smart cities. On the one hand, the use of deep learning techniques on data retrieved from multiple IoT-based sources in a smart city can enable the prediction of relevant patterns about the functions and the pace of change in the city. On the other hand, images captured from different types of cameras (e.g., surveillance cameras, mobile phones, etc.) can be employed to transform visual data into insightful information which can in turn be used for enhancing the existing deep learning models. The manuscript "Urban management image classification approach based on deep learning" by Kang and Ding proposes a smart city management platform to process images reported by citizens through their mobile phones so as to locate problems in urban scenarios. In order to cope with issues such as low resolution and complex-background images, the authors propose the use of an improved convolutional neural network to design an algorithm named ZCNN which automatically classifies images into several categories of meaningful urban items such as "car" or "rubbish". The algorithm is based on a unified ZCA (Zero-phase Component Analysis) processing method that facilitates efficient image analysis. Experiments performed with more than 5,000 images demonstrate that the proposed platform can identify illegal car parking or waste items outside of garbage bins, outperforming other techniques.

The combination of DL and VA techniques can also be applied to maritime surveillance. Thus, the manuscript "Machine learning-based ship detection and tracking using satellite images for maritime surveillance" by Wang et al. introduces a novel ship detection and tracking (SDT) process to remotely monitor ships through satellite images. First, a filtering algorithm is developed to remove the noise from the satellite image, then a dynamic sea-land segmentation technique is applied, and finally the ships are identified using various machine learning techniques such as Support Vector Machine and Random Forest, among others. In order to validate their proposal, the authors evaluate a dataset with 4,000 satellite images of the San Francisco Bay and San Pedro Bay areas (California), showing the Support Vector Machine model as the best option for this task with an accuracy of $95 \%$.

Another application of this combination of deep learning and image processing can be found in the area of Ambient Assisted Living (AAL). In their manuscript "A novel computer vision-based data driven modelling approach for person-specific fall detection", Gong et al. explore the use of a regular RGB camera for recording the daily activities of an elderly person at home. The human body silhouette is then extracted from these recordings and fed to a convolutional neural network auto-encoder (CNN-AE) so as to automatically identify the representative features 
out of the silhouette. These features are used to train a one-class Support Vector Machine (OCSVM) to detect the occurrence of falls. The model obtained is person-specific, that is to say, it takes into account the information about the specific living environment and typical activities of the person being monitored to achieve better performance. The system is evaluated through a simulation scenario involving a 75-year-old person performing 25 activities and 14 falls which produce 3,171 frames to be analyzed. The results show an accuracy of about $95 \%$ with a low false alarm rate.

This issue ends with the manuscript "RECITE: A framework for user trajectory analysis in cultural sites" by Orenes-Vera et al., who propose a framework for the classification of tourist trajectories in cultural exhibitions such as museums. The framework gathers data about a visitor's activities by means of a Bluetooth Low Energy (BLE) infrastructure to track the visitor's indoor position and monitor the museum's guidance device used by the visitor to know the multimedia resources being consumed. This combined information is used as input to an ensemble of fuzzy rule models to classify the visitor's activity in real time. The classifier tags the visitor in categories such as "observer", "interested", "in hurry" or "lost", which may help the operators of the exhibits to offer a more personalized service. The framework is evaluated in two main rooms of a museum connected by a corridor involving 15 BLE beacons and ad-hoc applications installed on a guidance device and shows an accuracy above $90 \%$ for all the various types of trajectories considered.

\section{Upcoming issues}

The following is a list of upcoming issues of JAISE:

- November 2021: Regular Issue.

- January 2022: Thematic Issue on Sensing and Computing for Smart Healthcare.

- March 2022: Regular Issue.

- May 2022: Thematic Issue on Secure and Advanced Technology for Intelligent Environments.

- July 2022: Regular Issue.

- September 2022: Thematic Issue on Current Trends and the Future of Internet of Things in Industry and Enterprise.

More information on the call for papers to the future thematic issues is available on the webpage of JAISE at: http://www.iospress.nl/journal/journalof-ambient-intelligence-and-smart-environments/. 